Subscriber access provided by Queen Mary, University of London

Article

\title{
Effects of High Pressure on Phospholipid Bilayers
}

Wei Ding, Michail Palaiokostas, Ganesh Shahane, Wen Wang, and Mario Orsi

J. Phys. Chem. B, Just Accepted Manuscript • DOI: 10.1021/acs.jpcb.7b07119 • Publication Date (Web): 19 Sep 2017

Downloaded from http://pubs.acs.org on September 20, 2017

\section{Just Accepted}

"Just Accepted" manuscripts have been peer-reviewed and accepted for publication. They are posted online prior to technical editing, formatting for publication and author proofing. The American Chemical Society provides "Just Accepted" as a free service to the research community to expedite the dissemination of scientific material as soon as possible after acceptance. "Just Accepted" manuscripts appear in full in PDF format accompanied by an HTML abstract. "Just Accepted" manuscripts have been fully peer reviewed, but should not be considered the official version of record. They are accessible to all readers and citable by the Digital Object Identifier (DOI®). "Just Accepted" is an optional service offered to authors. Therefore, the "Just Accepted" Web site may not include all articles that will be published in the journal. After a manuscript is technically edited and formatted, it will be removed from the "Just Accepted" Web site and published as an ASAP article. Note that technical editing may introduce minor changes to the manuscript text and/or graphics which could affect content, and all legal disclaimers and ethical guidelines that apply to the journal pertain. ACS cannot be held responsible for errors or consequences arising from the use of information contained in these "Just Accepted" manuscripts. 


\title{
Effects of High Pressure on Phospholipid Bilayers
}

\author{
Wei Ding, ${ }^{\dagger}$ Michail Palaiokostas, ${ }^{\dagger}$ Ganesh Shahane, ${ }^{\dagger}$ Wen Wang, ${ }^{\dagger}$ and Mario \\ Orsi $^{*, \ddagger}$ \\ $\dagger$ School of Engineering \& Materials Science, Queen Mary University of London, Mile End \\ Road, London E1 $4 N S, U K$ \\ $\ddagger$ Department of Applied Sciences, University of the West of England, Coldharbour Lane, \\ Bristol BS16 1QY, UK \\ E-mail: Mario.Orsi@uwe.ac.uk
}

\begin{abstract}
The response of lipid membranes to changes in external pressure is important for many biological processes, and it can also be exploited for technological applications. In this work, we employ all-atom molecular dynamics simulations to characterize the changes in the physical properties of phospholipid bilayers brought about by high pressure (1000 bar). In particular, we study how the response differs, in relation to different chain unsaturation levels, by comparing mono-unsaturated 1-palmitoyl2-oleoyl-phosphatidylcholine (POPC) and bi-unsaturated dioleoyl-phosphatidylcholine (DOPC) bilayers. Various structural, mechanical and dynamical features are found to be altered by the pressure increase in both bilayers. Notably, for most properties, including bilayer area and thickness, lipid order parameters, lateral pressure profile, and curvature frustration energy, we observe significantly more pronounced effects for mono-unsaturated POPC than bi-unsaturated DOPC. Possible biological implications of the results obtained are discussed, especially in relation to how different lipids can control the structure and function of membrane proteins.
\end{abstract}




\section{Introduction}

Pressure is one of the most important environmental factors for living organisms; growing attention is being devoted especially to the effects of high pressure on biological processes. On the one hand, experimental studies have revealed how various organisms and tissues undergo cell damage under high pressure. ${ }^{1-3}$ On the other hand, some bacteria and viruses have been shown to be pressure-tolerant. ${ }^{4,5}$ Barophilic organisms have been discovered in deep marine environments where the hydrostatic pressure can reach 1100 bar (110 MPa), a factor of over one thousand greater than atmospheric pressure; ${ }^{6}$ in general, it is of great scientific interest to understand how life can adapt to such extreme environmental pressures. ${ }^{7}$ To study the biological impact of high pressure, researchers have investigated a range of phenomena related to pressure change, such as the inactivation of pathogens ${ }^{8}$ or the denaturation of proteins. ${ }^{9}$ High hydrostatic pressure is also relevant to the action mechanism of anesthetics, ${ }^{10}$ and it can be exploited for protein crystallization ${ }^{11}$ as well as for drug encapsulation and delivery. ${ }^{12,13}$ Moreover, the food industry has seen in recent years the emergence of pressure-based processing technologies, for example as an approach to improve pasteurization. Compared to traditional high-temperature treatments, high-pressure food processing is expected to better preserve the original organoleptic properties such as flavor and color, as well as the nutritional content. ${ }^{14,15}$

The lipid bilayer membrane, a key structural and functional component of cells, is regarded as one of the most pressure-sensitive biological systems. ${ }^{16}$ Various aspects of the lipid bilayer have been observed to be altered under high pressure conditions, such as volume, fluidity, and phase transition. ${ }^{17-21}$ Moreover, the folding/unfolding and aggregation processes of membrane-embedded proteins, who closely interact with membrane lipids, can also be affected by pressure changes. ${ }^{22,23}$ Overall, pressure effects on lipid membranes appear to be of great importance for living organisms. As a remarkable example, many bacteria have been found to modify their membrane lipid composition in response to environmental changes in hydrostatic pressure. ${ }^{24}$ In particular, evidence collected over the past decades on deep 
sea organisms suggests that the increased deep sea hydrostatic pressure selects for a higher ratio of unsaturated fatty acids in the lipid membrane. ${ }^{25-28}$ Unsaturated fatty acyl chains in membrane phospholipids are characterized by one or more double bonds, which induce more disordered conformations, ultimately maintaining membrane fluidity (an essential condition for most biological functions) even under high pressure. In particular, it has been shown experimentally that a bi-unsaturated 16:0-18:2 PC (PLPC) bilayer shows greater resistance to pressure-induced liquid-to-gel phase transition than a mono-unsaturated 16:0-18:1 PC (POPC) bilayer. ${ }^{17}$

While previous experimental work has shed light on pressure-induced structural and energetic changes in relation to the unsaturation level in lipid membranes, molecular-level mechanisms are still not well understood. In practice, measuring microscopic membrane properties while simultaneously maintaining the pressure at a desired level is fraught with great technical challenges. ${ }^{18}$

As an alternative to conventional experiments, molecular simulation represents a powerful tool to study pressure effects on lipid membrane systems, thanks to the ability to accurately control the simulated pressure while obtaining quantitative nanoscale measurements. ${ }^{29-31}$ In this work, we conduct atomistic molecular dynamics simulations to study the effects of high pressure on key physical properties of lipid bilayers. In particular, we focus on two lipid types that are prevalent in biological membranes, i.e., dioleoyl-phosphatidylcholine (DOPC) and 1-palmitoyl-2-oleoyl-phosphatidylcholine (POPC). These two lipids are very similar apart from a key difference in unsaturation content, so that their response to pressure changes will also allow us to investigate the specific role of unsaturation in determining related changes in physical properties. Specifically, DOPC has two unsaturated chains each containing a C-C double bond, whereas POPC has one such mono-unsaturated chain (as DOPC) while the other chain is fully saturated (all C-C linkages are single bonds). By comparing the changes induced by increasing the pressure on bilayers comprising these two representative lipid types, we aim to provide a systematic understanding of the role that unsaturation plays 
in the membrane response to increased pressures. In particular, we simulate the two bilayer systems at the standard atmospheric condition of 1 bar and at the higher pressure level of 1000 bar, under which both the DOPC and POPC bilayers still remain in the fluid $L_{\alpha}$ phase (which is the biologically relevant phase). At each of these two pressures, a series of structural, dynamical, mechanical, and electrostatic properties are measured and compared. While research on lipid bilayers at standard atmospheric condition has been extensive, to our knowledge this is the first simulation study investigating high pressure effects on bilayer properties using fully atomistic models. The few previous simulations of lipid bilayers at high pressure were conducted with either united-atom or coarse-grained models. ${ }^{32,33}$ In this work, we simulate all-atom models to investigate fundamental bilayer properties including lipid area, lipid volume, bilayer thickness, lipid lateral diffusivity, and deuterium order parameters. Notably, we also calculate the lateral pressure and dipole potential, two elusive and often overlooked trans-membrane profiles which have been suggested to play key roles in numerous membrane phenomena. ${ }^{34-36}$ The lateral pressure (or stress) profile represents the distribution of lateral stresses as a function of depth inside the bilayer. Experimentally, given the nanoscopic thickness of a typical lipid bilayer membrane, it is extremely difficult to measure internal stresses. Attempted measurements suggest significant depth-dependent variation, ${ }^{37,38}$ and computational modeling studies have indeed quantified lateral pressure changes on the order of several hundred bars. ${ }^{39}$ Naturally, substantial mechanical influence on embedded molecules such as membrane proteins or permeants is expected as a consequence of the existence of the lateral pressure profile. ${ }^{40-43}$ Moreover, the lateral pressure profile underpins a number of elastic parameters, such as the spontaneous curvature and the bending rigidity, which are critical indicators of the mesoscopic phase behavior of a hydrated lipid bilayer. ${ }^{18,19,41}$ For example, changes in the lateral pressure profile and related elastic properties may lead to the destabilization of cell membranes. ${ }^{44-46}$ The dipole (electrostatic) potential profile, arising from the preferential alignment of the dipole moments of water molecules and dipolar segments of lipid molecules, is another trans-membrane property that 
is of great biological relevance yet also very difficult to probe by experiment. ${ }^{35,47}$ In this study, we investigate the sensitivity of DOPC and POPC bilayers to external pressure by evaluating the dipole potential profile as a function of depth across the bilayers.

\section{Methods}

\section{Lipid bilayers}

Two single-type lipid bilayer systems have been investigated. One system comprises 1palmitoyl-2-oleoyl-phosphatidylcholine (POPC) lipids, which are mono-unsaturated. The other system comprises dioleoyl-phosphatidylcholine (DOPC) lipids, which are bi-unsaturated. The molecular structures of the two lipid types are shown in Figure 1. Each bilayer sys-
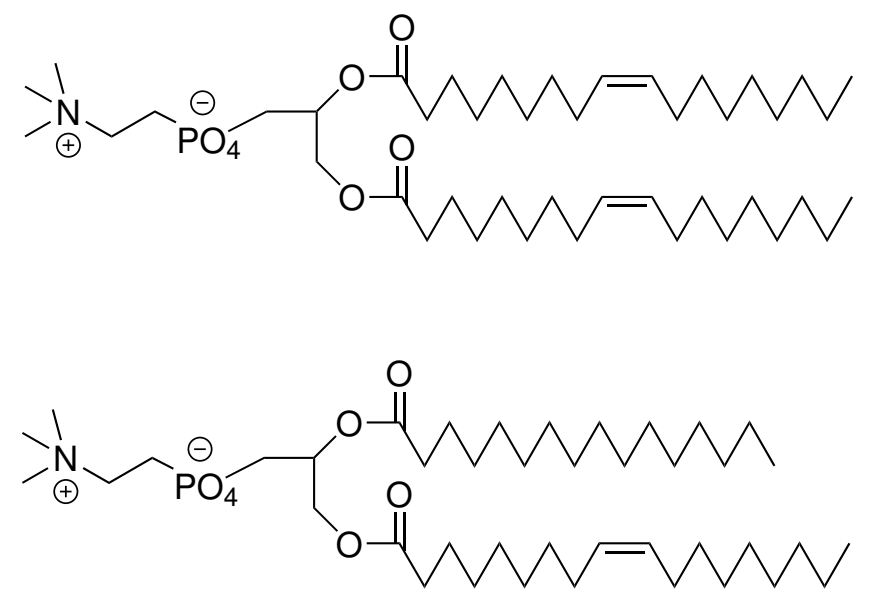

Figure 1: Molecular structures of DOPC (above) and POPC (below).

tem contains 128 lipid molecules and is fully hydrated with 4300 water molecules (with a water/lipid ratio of approximately 33.6). ${ }^{48,49}$ Initial pre-equilibrated configurations were obtained from our previous studies. ${ }^{50}$

\section{Simulation details}

The bilayer systems were modelled with the CHARMM36 all-atom force field ${ }^{48}$ and simulated with the Gromacs 5.1 software. ${ }^{51}$ Long range electrostatic interactions were solved by 
the PME (Particle-Mesh Ewald) method. ${ }^{52}$ Bonds and angles in water molecules were constrained by the SETTLE algorithm; ${ }^{53}$ the LINCS algorithm ${ }^{54}$ was used to constrain all other hydrogen-related bonds, with a numerical expansion up to fourth order and two iterations in every step for correcting rotational effects. With these constrained algorithms applied, the systems' total energy was well conserved at the selected timestep of 2 fs. Each of the DOPC and POPC bilayers were simulated at two pressure levels, 1 bar and 1000 bar, respectively. The Parrinello-Rahman barostat method was used, ${ }^{55}$ with the pressure coupled semi-isotropically (isotropic in the two directions along the bilayer plane but independent to the one in the normal direction) and a coupling time constant of 1 ps. The temperature was maintained at $310 \mathrm{~K}$ for all systems, by applying the velocity-rescale thermostat ${ }^{56}$ with a damping time constant of 0.1 ps. Each simulation was run for $1000 \mathrm{~ns}$ in total. The first 200 ns were regarded as equilibration and the following $800 \mathrm{~ns}$ were used for data analysis. Data for all properties were sampled every 50 ps.

It is relevant to note that the water model employed, TIP3P, has been previously simulated under high pressures up to 4000 atm, yielding results in reasonable agreement with experiment. ${ }^{57-59}$ In particular, referring to the density under an external pressure of 1000 atm as relevant to this work, it was calculated ${ }^{59}$ that TIP3P only slightly overestimates the experimental value, by $\sim 0.01 \mathrm{~g} \mathrm{~cm}^{-3}$. Regarding the self-diffusion coefficient, the TIP3P value has been shown previously ${ }^{58}$ to be consistently more than twice larger than the corresponding experimental data over a pressure range from 0 to 4000 atm. While this is in principle not ideal, no repercussions are expected for most of the calculations reported here, as in general it is well known that increased diffusion does not affect thermodynamic properties. The only property in this work that may be affected is the lipid lateral diffusion; however, since this property is mostly determined by lipid-lipid interactions rather than interaction with water, we expect any artefact due to increased water diffusion to be negligible. 


\section{Data analysis}

The following properties were evaluated: area per lipid $\left(A_{L}\right)$, volume per lipid $\left(V_{L}\right)$, bilayer thickness $\left(d_{H H}\right)$, lateral diffusion coefficient $\left(D_{L}\right)$, deuterium order parameter $\left(S_{C D}\right)$, electron density profile $(\rho(z))$, dipole potential profile $(\Psi(z))$, lateral pressure profile $(\Pi(z))$, monolayer spontaneous curvature $\left(c_{0}^{m}\right)$, monolayer bending modulus $\left(\kappa^{m}\right)$, curvature frustration energy $\left(\kappa^{m} c_{0}^{m}\right)$. Calculations of these properties were carried out following standard approaches, as detailed previously. ${ }^{60,61}$ To estimate the average volume per lipid $V_{L}$, two extra simulations consisting of 4300 TIP3P water molecules (same amount as in the bilayer systems) in the bulk liquid phase were performed at 1 bar and 1000 bar, respectively, so that $V_{L}$ could be computed as $V_{L}=\left(V_{\text {total }}-V_{\text {water }}\right) / 128$, where $V_{\text {total }}$ is the total volume of the bilayer systems and $V_{\text {water }}$ is the volume of the bulk water simulations. Regarding the lateral pressure profile, a modified version of Gromacs, the Gromacs-LS package, ${ }^{62,63}$ was used to rerun the simulation trajectories and to output local stress tensors. Since the long-range electrostatic solver is not implemented in the Gromacs-LS package, an increased cut-off distance of $2.2 \mathrm{~nm}$ was used for the Coulomb interactions, as recommended by the package developers. ${ }^{62}$

Regarding the statistical analysis, uncertainties for all calculated properties (except for $\left.D_{L}\right)$ were estimated by the block averaging procedure, ${ }^{64,65}$ with a block size of $40 \mathrm{~ns}$. The only exception, $D_{L}$, was obtained from the linear fitted slope of the averaged 2-dimensional mean squared displacement (MSD) following a standard procedure. ${ }^{59,66}$ The MSD was treated by the time-averaged method, with the initial reference point reset every 40 ns. ${ }^{60,65}$ All numerical results will be presented in the format "mean \pm standard error (S.E.)" unless otherwise specified.

To rigorously compare the results obtained for the two different lipid types at the two levels of external pressure studied, we carried out standard two-sample t-tests wherever appropriate; a difference between two means is considered statistically significant if the corresponding $p$ value is less than 0.05 , as per predominant convention. 


\section{Results and discussions}

\section{Structural properties}

The area per lipid $\left(A_{L}\right)$, volume per lipid $\left(V_{L}\right)$, and bilayer thickness $\left(d_{H H}\right)$ were calculated for the DOPC and POPC bilayer simulations at the two investigated pressures. Results are summarized in Table 1. Regarding the values obtained at atmospheric pressure, our results are

Table 1: Structural properties for the simulated bilayer systems

\begin{tabular}{lccc}
\hline & $\begin{array}{c}A_{L} \\
\left(\AA^{2}\right)\end{array}$ & $\begin{array}{c}V_{L} \\
\left(\AA^{3}\right)\end{array}$ & $\begin{array}{c}d_{H H} \\
(\AA)\end{array}$ \\
\hline DOPC, 1 bar & $68.72 \pm 0.09$ & $1290.46 \pm 0.09$ & $38.42 \pm 0.05$ \\
DOPC, 1000 bar & $65.43 \pm 0.12$ & $1235.91 \pm 0.06$ & $38.28 \pm 0.06$ \\
POPC, 1 bar & $65.10 \pm 0.09$ & $1243.04 \pm 0.26$ & $38.74 \pm 0.03$ \\
POPC, 1000 bar & $60.80 \pm 0.13$ & $1188.18 \pm 0.55$ & $39.14 \pm 0.07$ \\
\hline
\end{tabular}

A t-test was performed for each pair of values from the same bilayer between the two pressures. Statistically significant differences $(p<0.05)$ exist for each pair, with the only exception of $d_{H H}$ for the DOPC bilayer $(p=0.13)$.

consistent with previous reports from both experiments and atomistic simulations. ${ }^{48,50,67-69}$ A comparison with the results obtained at the high pressure of 1000 bar reveals noticeable effects. In particular, for the area per lipid $A_{L}$, a significant reduction is shown for both POPC and DOPC as the pressure increases from 1 bar to 1000 bar. Comparing the extent to which $A_{L}$ changes, the reduction for POPC (an average $4.30 \AA^{2}$ or $7.4 \%$ decrease) is larger than that observed for DOPC (an average $3.29 \AA^{2}$ or $4.7 \%$ decrease). Regarding the lipid volume, increasing the pressure causes a reduction of $V_{L}$ for both lipid types, as intuitively expected, with an average $54.5 \AA^{3}$ (4.2\%) decrease being shown for DOPC and $54.9 \AA^{3}(4.4 \%)$ for POPC. Regarding the bilayer thickness $\left(d_{H H}\right)$, the pressure increase does not induce a significant effect on DOPC. However, it is possible to identify a statistically significant increase for POPC, albeit small (0.4 $\AA$ or 1\%). While the change seems negligible, previous findings indicate that membrane protein channels can be deformed and inactivated by hydrophobic mismatch even for small changes in bilayer thickness of less than $1 \AA{ }^{70}$ Previous studies on the relation between bilayer thickness and pressure have given varying 
results by different techniques and measurements: a recent experimental work observed that the lattice parameter of DOPC vesicle increased along with the pressure increase, ${ }^{12}$ while insensitivity of $d_{H H}$ was found for DPPC bilayers in the fluid phase at different pressures in a coarse-grained simulation study. ${ }^{33}$

Electron density profiles $(\rho(z))$ for both bilayers are shown in Figure 2. It can be seen

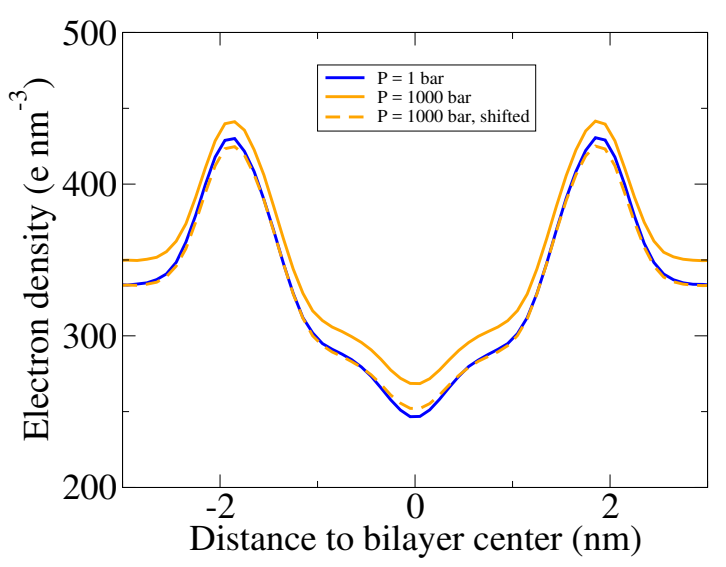

(a)

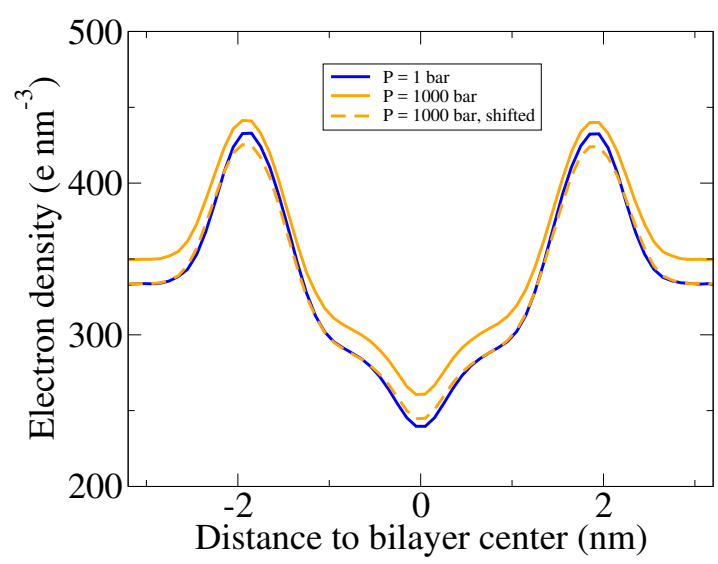

(b)

Figure 2: Electron density for (a) DOPC and (b) POPC bilayers at 1 bar (blue) and 1000 bar (orange). To better highlight differences within each membrane type (see main text), the dashed orange lines represent electron density profiles at 1000 bar that have been shifted to overlap those at 1 bar in the water phase. Error bars are smaller than the thickness of the lines.

that $\rho(z)$ for both DOPC and POPC increases across the whole depth $z$ as the pressure is increased from 1 bar to 1000 bar, consistently with the reduction in lipid area and volume reported earlier. To better highlight differences within each membrane type, the electron density profiles at 1000 bar have also been shifted so that they overlap those at 1 bar in the water phase; such shifted curves can be seen in Figure 2 as dashed orange lines. Compared to the 1 bar curves, it can be seen that the 1000 bar shifted profiles are slightly lower at the two peaks corresponding to the lipid head groups while higher at the central trough. These differences indicate that most of the volume reduction takes place in the bilayer center (the lipid tail end region). This can be interpreted as a relatively higher resistance to volume 
reduction in the lipid head groups, since this region is already the densest; conversely, the bilayer is compressed more markedly in the central core, where the density is lowest (thus corresponding to highest free volume). It is relevant to note that, despite the compression brought about by the pressure increase, no chain interdigitation is observed in any of the systems studied, as indicated by the well-defined central minima in all the electron density diagrams of Figure 2. Further confirmation of the absence of interdigitation can be found in the Supporting Information, where we report individual electron density profiles for only the chain components (Figure S1).

\section{Deuterium order parameters}

Deuterium order parameters $\left(S_{C D}\right)$ for acyl-chain carbon atoms computed from each investigated system are displayed in Figure 3. The $S_{C D}$ values for both chains in DOPC lipids (Figure 3a and 3c) and the sn-2 chain in POPC lipids (Figure 3d) exhibit generally similar features, in that the $S_{C D}$ value drops to a minimum at positions corresponding to the double-bonded atoms. In contrast, the POPC sn-1 chain (Figure 3b) exhibits a monotonically decreasing $S_{C D}$ from the position corresponding to the glycerol segment to the end of the chain, as is normal for a fully-saturated chain.

As intuitively expected, higher pressure causes the $S_{C D}$ values to increase, reflecting enhanced order brought about by a reduction in free volume (consistently with the results on the lipid volume reported earlier). Specifically, the increase in order parameters indicates a change in orientation of the corresponding chain segments, which tend to become more aligned to the direction perpendicular to the bilayer plane. By comparing $s n-2$ (unsaturated) tails in DOPC and POPC (Figure 3c and Figure 3d), different changes induced by the pressure increase can be noticed. The effect on POPC is more pronounced, with an average $S_{C D}$ increase of 0.015 compared with 0.006 for its DOPC counterpart. Although not directly comparable, $S_{C D}$ values for the POPC sn-1 chain are increased markedly by 0.020 , while the increase for DOPC sn-1 chain is 0.007. Experimental studies have suggested that $S_{C D}$ 


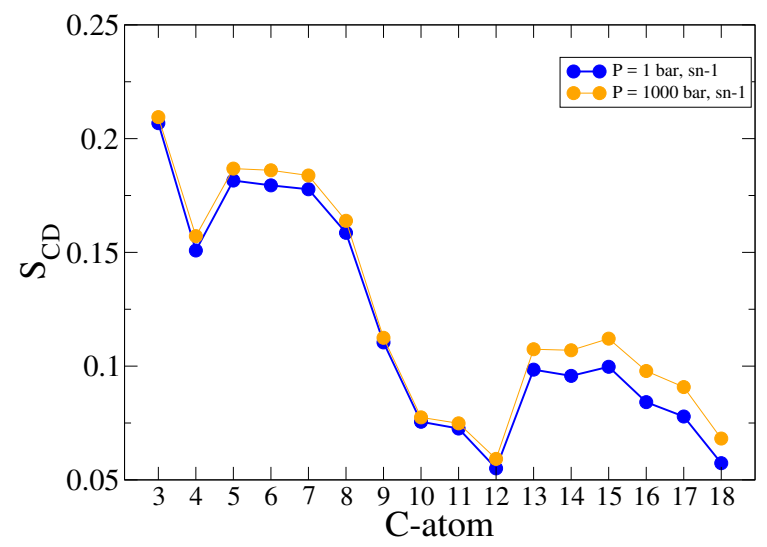

(a)

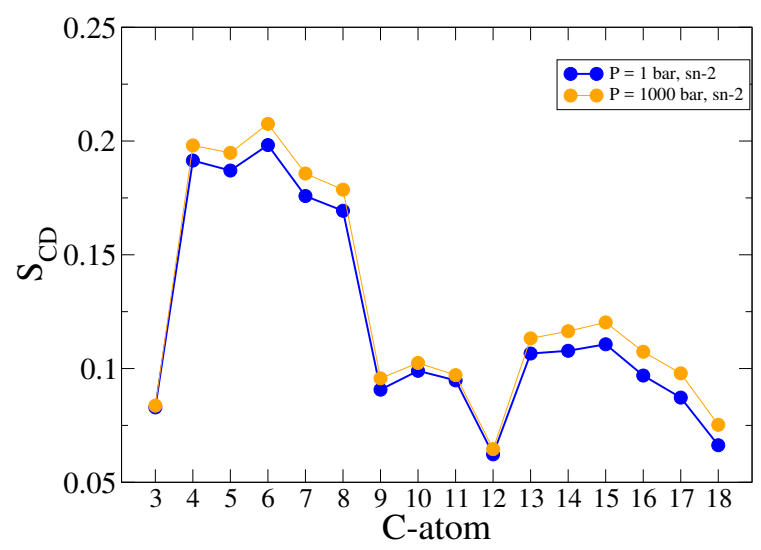

(c)

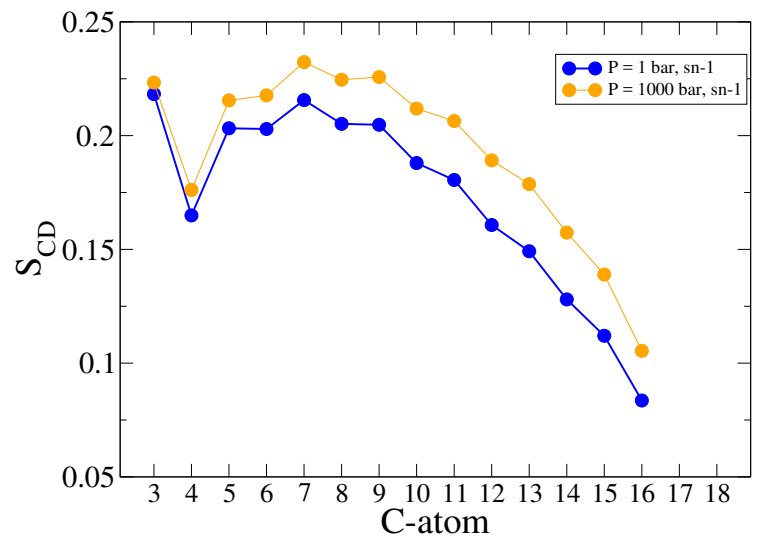

(b)

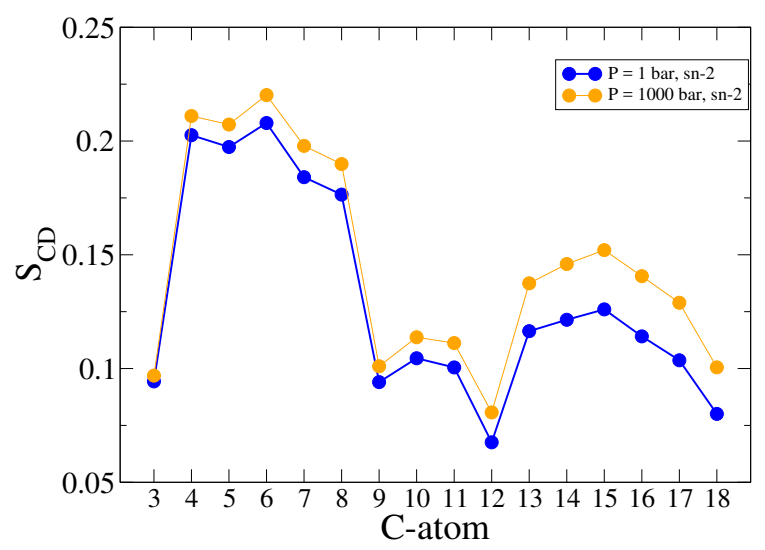

(d)

Figure 3: $S_{C D}$ for (a) DOPC, $s n-1$ chain, (b) POPC, $s n$-1 chain, (c) DOPC, $s n-2$ chain, and (d) POPC, sn-2 chain at 1 bar (blue) and 1000 bar (orange). Error bars are smaller than the symbols' size. 
values for POPC in the $L_{\alpha}$ phase increase by roughly $10^{-4}$ per MPa (10 bar) ${ }^{71}$ at $300 \mathrm{~K}$, corresponding to 0.01 over 1000 bar, which is in reasonable agreement with our results.

Overall, our comparison between $S_{C D}$ order parameters in DOPC and POPC shows that the less saturated POPC bilayer is more sensitive to the pressure increase, consistently with our earlier observation on lipid area, lipid volume, and bilayer thickness.

In general, the changes in structural properties from our simulations are consistent with previous theoretical understanding that increased unsaturation (as in DOPC vs. POPC) results in a stronger resistance to structural change under high pressure. ${ }^{17}$

\section{Lateral diffusion}

The lateral diffusion coefficient $\left(D_{L}\right)$ measures the long range motion of lipids in the bilayer plane. The $D_{L}$ values obtained from our DOPC and POPC simulations at 1 bar are $14.7 \pm$ $0.8 \mathrm{~nm}^{2} / \mu s$ and $16.2 \pm 0.6 \mathrm{~nm}^{2} / \mu s$, respectively. These results are in reasonable agreement with previously reported values for phospholipid bilayers from both experiments and simulations. ${ }^{61,67,72,73}$ Comparing the two lipid types, we find no statistically significant difference between $D_{L}$ for DOPC and POPC at 1 bar $(p=0.18)$; this is in agreement with the earlier

conclusion by Ollila et al. ${ }^{74}$ that unsaturation does not influence the lateral diffusivity of lipids. Under the high pressure of 1000 bar, we obtain $D_{L}$ values of $9.8 \pm 1.5 \mathrm{~nm}^{2} / \mu \mathrm{s}$ and $7.1 \pm 0.2 \mathrm{~nm}^{2} / \mu \mathrm{s}$ for DOPC and POPC, respectively. It is clear that, for both lipid types, there is a substantial reduction in lateral diffusion brought about by the pressure increase; this effect is expected intuitively, and is consistent with the observed reduction in system volume. Comparing the $D_{L}$ values for the two lipid types at 1000 bar, no statistically significant difference is detected $(p=0.053)$, similarly to what was found for the systems at 1 bar. In summary, while high pressure markedly reduces the lateral diffusivity for both the DOPC and POPC bilayers, our results do not show specificity to the lipid type. 


\section{Lateral pressure profile}

The lateral pressure profile $(\Pi(z))$ characterizes the inhomogeneous and depth-dependent distribution of lateral stresses across the bilayer. The calculation of $\Pi(z)$ from molecular simulations provides nanoscale insights for the understanding of the mechanical stability of the membrane itself as well as the interactions between lipids and other biomembrane constituents (such as membrane proteins). The $\Pi(z)$ profiles computed for the systems investigated in this work are shown in Figure 4. Regarding the profiles at 1 bar, both the

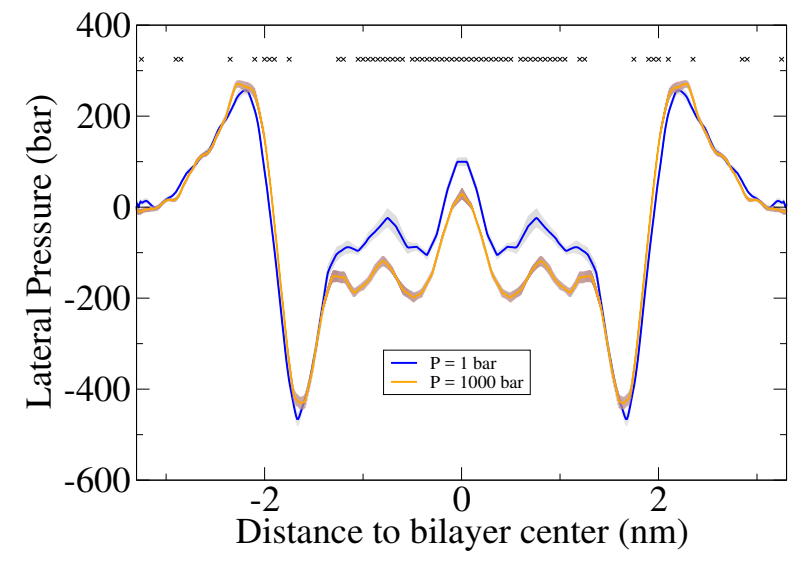

(a)

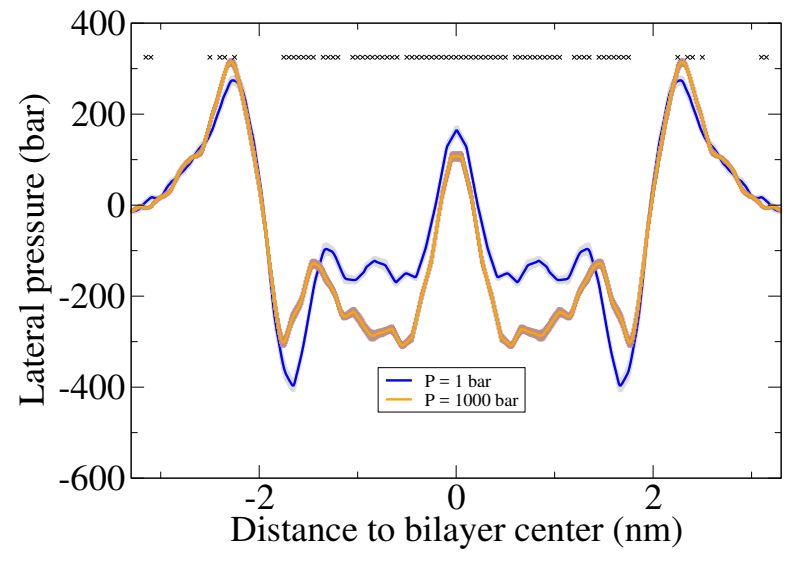

(b)

Figure 4: $\Pi(z)$ for (a) DOPC and (b) POPC bilayers at 1 bar (blue) and 1000 bar (orange). Shaded areas indicate the statistical uncertainty (mean \pm S.E.). Statistical analysis was performed to compare the difference between the two curves in each diagram. The stars at the top of each diagram indicate the positions where the values of the two curves are statistically different $(p<0.05)$.

DOPC and POPC results are consistent with previous literature. ${ }^{39,75,76}$ In particular, large positive peaks of $\sim 300$ bar can be seen at a distance of $\sim 2 \mathrm{~nm}$ to the bilayer center, reflecting net repulsive forces primarily attributable to steric and electrostatic interactions between lipid heads and water. Proceeding deeper inside the bilayers, at a depth corresponding roughly to the glycerol groups, we can observe sharp negative troughs, which are typically explained in terms of attractive forces due to the hydrophobic effect. ${ }^{41,77}$ The bilayer center is instead characterized by pressure peaks that are believed to originate from loss of entropy 
in the chain region. ${ }^{78,79}$

Regarding the effect of increased external pressure, in the outer regions corresponding to the water phase and headgroup layer no significant differences are observed for both lipid types. Such a low sensitivity of $\Pi(z)$ to external pressure changes in these regions has been predicted theoretically on the basis of the relatively high local density ${ }^{18}$ (which can be seen in the electron density profiles of Figure 2). Deeper inside the bilayer, the large hydrophobic troughs exhibit a response to high pressure which is dependent on the lipid type. In particular, increased external pressure does not significantly alter the DOPC hydrophobic troughs, whereas the POPC troughs markedly decrease in magnitude, by $\sim 100$ bar. Regarding the lipid chain region, it can be seen that the increase in external pressure brings about a significant and substantial decrease in $\Pi(z)$ for both lipid types. This effect can be correlated to the increase in chain order parameters under high pressure observed earlier (Figure 3). Such a relation was also found in previous studies focusing on other external factors like temperature and chain length. ${ }^{74,80}$ From our data, it can be seen that the pressure-induced changes are more prominent for POPC, consistently with the larger increase in $S_{C D}$ values with respect to DOPC (as discussed earlier). In particular, taking the chain region to be within $1.3 \mathrm{~nm}$ from the bilayer center, we observe an average lateral pressure drop of $82.8 \pm 19.2$ bar for DOPC under high pressure, while for POPC we calculate a larger drop of $101.7 \pm 35.9$ bar.

Overall, it is evident that the lateral pressure profile for both DOPC and POPC bilayers is affected by the external pressure, and that the effects are more pronounced for the mono-unsaturated POPC compared to the bi-unsaturated DOPC. Higher lipid unsaturation therefore reduces bilayer sensitivity to increased hydrostatic pressure; this is analogous to the behavior observed previously for a number of structural properties. Further quantitative analysis of the lateral pressure profiles, based on elastic parameters that can be derived via numerical integration, is reported in the following section. 


\section{Elastic properties}

Key elastic parameters of the bilayer are connected analytically with $\Pi(z)$. Specifically, the product of the monolayer bending modulus $\kappa^{m}$ and the monolayer spontaneous curvature $c_{0}^{m}$ is equal to the fist integral moment of $\Pi(z)$ :

$$
\kappa^{m} c_{0}^{m}=\int_{0}^{l} z \Pi(z) \mathrm{d} z
$$

where $z=0$ at the center of the bilayer and $z=l$ in the water phase. ${ }^{81,82}$ To obtain the first integral moment of $\Pi(z)$ (corresponding to the right hand side of equation 1 ), a conventional numerical integration was performed. The monolayer bending modulus $\kappa^{m}$ was estimated by the relation $\kappa^{m}=k_{A}\left(d_{H H}-10\right)^{2} / 48$ based on an empirical model. ${ }^{83}$ The monolayer spontaneous curvature $c_{0}^{m}$ can then be easily obtained as the ratio between the first integral moment of $\Pi(z)$ and the monolayer bending modulus $\kappa^{m}$. The values obtained for these elastic properties at the studied pressures for each bilayer are presented in Table 2.

Table 2: Elastic properties for the simulated bilayer systems

\begin{tabular}{lccc}
\hline & $\begin{array}{c}\kappa^{m} \\
\left(10^{-20} \mathrm{~J}\right)\end{array}$ & $\begin{array}{c}c_{0}^{m} \\
\left(\mathrm{~nm}^{-1}\right)\end{array}$ & $\begin{array}{c}\kappa^{m} c_{0}^{m} \\
\left(10^{-21} \mathrm{Jm}^{-1}\right)\end{array}$ \\
\hline DOPC, 1 bar & $4.43 \pm 0.03$ & $-0.056 \pm 0.023$ & $-2.48 \pm 1.10$ \\
DOPC, 1000 bar & $3.92 \pm 0.03$ & $-0.091 \pm 0.034$ & $-3.55 \pm 1.44$ \\
POPC, 1 bar & $4.34 \pm 0.02$ & $-0.054 \pm 0.027$ & $-2.32 \pm 1.25$ \\
POPC, 1000 bar & $3.88 \pm 0.04$ & $-0.168 \pm 0.040$ & $-6.53 \pm 1.65$ \\
\hline
\end{tabular}

A t-test was performed for each property from the same bilayer type at the two pressures. Regarding $\kappa^{m}$, differences between the values at 1 bar vs. 1000 bar are significant $(p<0.05)$ for both bilayers. Regarding $c_{0}^{m}$, differences are significant for POPC but not for DOPC $(p=0.10)$. Regarding $\kappa^{m} c_{0}^{m}$, differences are again significant for POPC but not for DOPC $(p=0.55)$.

Our results for $\kappa^{m}$ for both bilayers at 1 bar are consistent with previous experimental and computational measurements. ${ }^{69,84,85}$ Regarding the effect of high pressure, our results show a slight decrease in $\kappa^{m}$ at the increased pressure of 1000 bar, for both bilayers. A recent experimental study ${ }^{12}$ on DOPC, which appears to be the first and so far only direct measurement of the bending rigidity of a lipid membrane under high pressure, shows a 
significant increase (up to a factor of 2) for external pressures of up to 400 bar. Interestingly however, for higher external pressures of up to 800 bar, the bending rigidity is then found to decrease down to the value observed at 1 bar.

For a symmetrical bilayer, the monolayer spontaneous curvature $c_{0}^{m}$ measures the intrinsic tendency of each of the monolayers to curl. Conventionally, the magnitude of $c_{0}^{m}$ quantifies a leaflet's desire to either curl towards the water phase in order to form inverse structures (for $c_{0}^{m}<0$ ) or to curl away from the water phase to form micellar structures (for $c_{0}^{m}>0$ ). When the magnitude of $c_{0}^{m}$ is small, the tendency to curl is not satisfied, and the monolayers assemble symmetrically to form a flat lamellar bilayer. Table 2 shows that, at 1 bar, both DOPC and POPC bilayers are characterized by small negative values of $c_{0}^{m}$, consistent with the well-known experimental behavior whereby these lipids form standard lamellar bilayer phases (as opposed to micelles or inverse phases); furthermore, the specific values obtained are in good agreement with previous literature. ${ }^{74,75,85,86}$ At the increased external pressure of 1000 bar, $c_{0}^{m}$ for POPC decreases (becomes more negative) whereas no statistically significant change is observed for DOPC (Table 2). The increased unsaturation level in DOPC with respect to $\mathrm{POPC}$ is therefore correlated with a resistance to changes in spontaneous curvature under high pressure. To our knowledge, no experimental measurement or other simulation study on the pressure dependence of the spontaneous curvature has been previously reported in the literature.

The quantity $\kappa^{m} c_{0}^{m}$ characterizes the membrane curvature frustration energy, also known as torque tension; it is a fundamental biophysical property that underlies mechanisms by which lipids can modulate the structure and function of membrane proteins. ${ }^{41,87,88}$ Our results are in fair agreement with available experimental measurements ${ }^{86}$ conducted at atmospheric pressure (1 bar), whereas no data are available at increased pressure. Considering the results obtained for our systems, we observe a statistically significant increase in the magnitude of $\kappa^{m} c_{0}^{m}$ under increased external pressure for POPC, whereas for DOPC there is no significant difference. It is interesting and relevant to compare our data with quantitative 
estimates, obtained theoretically by Cantor, ${ }^{87}$ of the changes in the product $\kappa^{m} c_{0}^{m}$ required to affect protein function. Specifically, from the analysis of collective rearrangements of simple geometric models of proteins, it was calculated ${ }^{87}$ that significant shifts in protein conformational equilibria could be induced by changes in $\kappa^{m} c_{0}^{m}$ of order $1.2 \times 10^{-21} \mathrm{~J} \mathrm{~nm}^{-1}$. From our data (Table 2), considering first DOPC, we can calculate that increasing the pressure from 1 to 1000 bar induces changes in $\kappa^{m} c_{0}^{m}$ of magnitude $1.1 \times 10^{-21} \mathrm{~J} \mathrm{~nm}^{-1}$, which is below Cantor's threshold, and anyway not statistically significant. For POPC however, the change in pressure brings about a substantially larger change in $\kappa^{m} c_{0}^{m}$ of magnitude $4.2 \times 10^{-21} \mathrm{~J} \mathrm{~nm}^{-1}$, which is well above Cantor's threshold, as well as being statistically significant. Therefore, at least in relation to Cantor's theoretical model, ${ }^{87}$ pressure-induced changes in $\kappa^{m} c_{0}^{m}$ would be large enough to alter protein conformational equilibria in a POPC bilayer, but not in a DOPC bilayer. Real systems are obviously much more complex, in that $\kappa^{m} c_{0}^{m}$ values will depend on the specific proteins as well as the lipid composition of the bilayer. Bearing this caveat in mind, our results nonetheless clearly suggest that the response in terms of $\kappa^{m} c_{0}^{m}$ values to a change in pressure can be highly sensitive to the lipid type, and specifically to the level of unsaturation. In particular, the mono-unsaturated POPC is found to be very sensitive to an increase in external pressure, with a much amplified effect on $\kappa^{m} c_{0}^{m}$ compared to the bi-unsaturated DOPC, for which the change with pressure is not significant.

\section{Dipole potential profile}

The electrostatic dipole potential profiles $\Psi(z)$ for the investigated DOPC and POPC bilayers at 1 bar and 1000 bar are shown in Figure 5. Interestingly, in this case, it can be seen that the change in pressure does not induce any substantial change in the dipole potential of either bilayer. The absence of pressure-induced effects was confirmed by further decomposition of $\Psi(z)$ into the individual contributions from water and lipid molecules (data not shown); these results are consistent with previous reports obtained under standard ambient pressure. ${ }^{50,89,90}$ Particularly, for both bilayers, the contribution due to water is largely positive, determines 


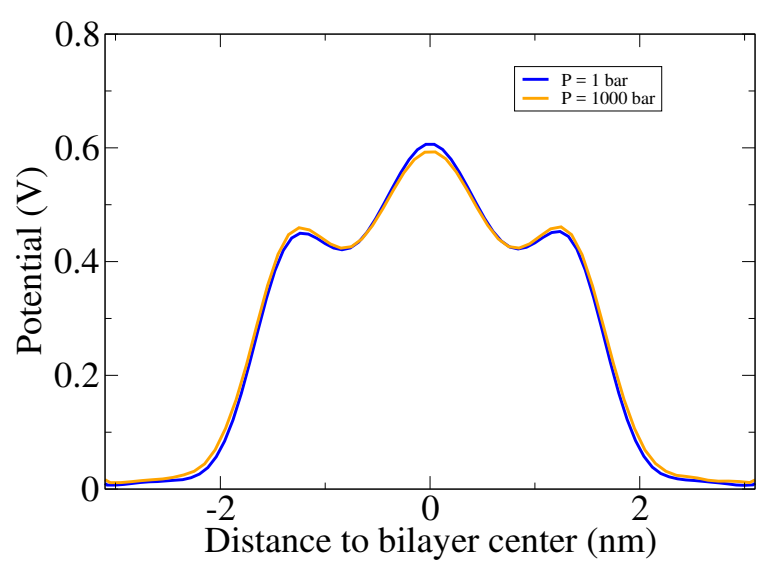

(a)

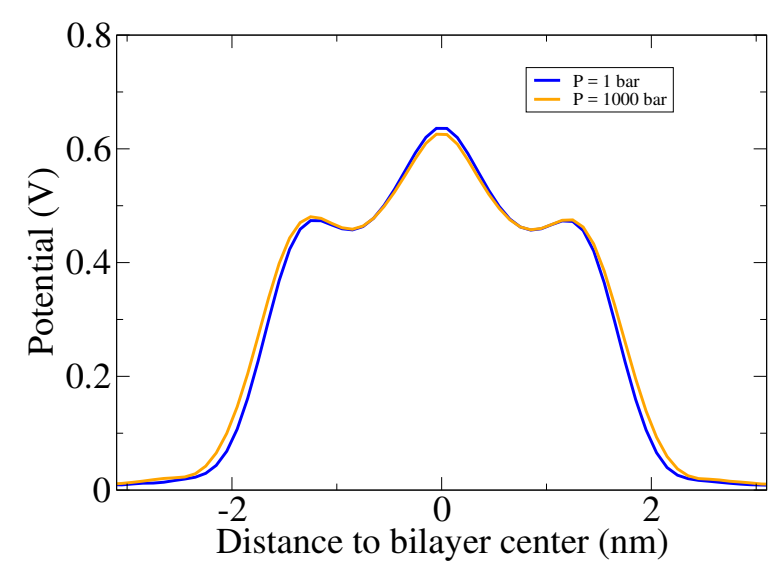

(b)

Figure 5: Dipole potential profiles for (a) DOPC and (b) POPC bilayers at 1 bar (blue) and 1000 bar (orange). Error bars are are smaller than the lines' thickness.

the sign of the overall profile, and is compensated by the negative lipid contribution. We are not aware of previously reported dipole potential data at other pressures, from either experiments or simulations. Overall, our results show that the dipole potential is not sensitive to changes in the external hydrostatic pressure, irrespectively of the type of lipid.

\section{Hydration and permeation}

To investigate more closely the interaction of the bilayers with water, we computed the individual electron density contribution for water (Figure S1 in the Supporting Information). The data obtained show that the response to the pressure change does not significantly alter the hydration features of the bilayer.

We also quantified the lipid headgroup orientation by calculating the angle between the lipid head P-N vector and the z-axis (i.e., the bilayer normal). The results obtained (reported in the Supporting Information) show that, for both the POPC and DOPC bilayers, the headgroup orientation does not change significantly in response to the imposed pressure increase. Incidentally, the absence of a significant effect on the headgroup orientation is consistent with the results obtained for the dipole potential, when one considers that the 
P-N vector is a major contributor to the overall dipole potential.

The lipid headgroups were also analyzed in terms of the average number of headgroupheadgroup and headgroup-water hydrogen bonds. Consistently with the electron density data on the headgroup hydration, no significant difference was found in the calculated hydrogen bond numbers upon increasing the external pressure (data reported in the Supporting Information).

To study the permeation of water through the bilayers, we calculated the number of water molecules crossing the bilayers during each of the $800 \mathrm{~ns}$ simulations analyzed. For POPC, the number of crossing events was $54 \pm 8$ at 1 bar and $14 \pm 2$ at 1000 bar, the difference being statistically significant $(p=0.04)$. For DOPC, the number of water crossings was $52 \pm 8$ at 1 bar and $33 \pm 3$ at 1000 bar; in this case the difference is not statistically significant $(p=0.15)$. Note that, to be able to estimate the standard errors, for each system we counted separately the crossings along the two directions aligned and anti-aligned the $z$ axis, thus obtaining two data values for each systems.

\section{Conclusions}

In this study, we employed fully atomistic molecular dynamics simulations to investigate the effects of a high external pressure of 1000 bar, compared to the ambient value of 1 bar, on a number of key physical properties of lipid bilayer systems. In particular, we studied and compared systems comprising either bi-unsaturated DOPC or mono-unsaturated POPC lipids. With the exception of the electrostatic dipole potential profile and headgroup hydration, the properties investigated were shown to be significantly affected by the pressure increase in both bilayers. Notably, for most properties the effects are amplified for the POPC bilayer, especially in relation to lipid area, chain order parameters, lateral pressure profile, spontaneous curvature and curvature frustration energy. Regarding the structural properties, our results provide molecular-level evidence to previous theoretical understanding 
that increased unsaturation results in a stronger resistance to structural change in response to high pressure. ${ }^{17}$ In particular, the existence of two unsaturated bonds (one in each tail) in the chemical structure of DOPC explains the less pronounced mechanical response to high pressure compared to the amplified response obtained for POPC, which features only one unsaturated bond. Moreover, the change in the curvature frustration energy $\kappa^{m} c_{0}^{m}$ was also much more pronounced for POPC; specifically, it was found to be significantly and substantially above a threshold value predicted to be required in order to affect the conformational equilibria of membrane-embedded proteins, ${ }^{87}$ whereas for DOPC we obtained a value below such threshold and not statistically significant. Thus it can be hypothesized that a change in external pressure as simulated in this work will induce a conformational change in specific proteins embedded in a mono-unsaturated POPC bilayer, while a biunsaturated DOPC bilayer would represent a control system where no conformational shift is expected; such hypothesis could be tested experimentally, for example, by considering channel proteins and measuring related permeation rates as response variables.

The lower unsaturation level in POPC with respect to DOPC seems therefore to make most physical properties of the corresponding bilayer more sensitive to high pressure. Conversely, increased unsaturation for DOPC correlates to higher resistance to changes in physical properties under increased pressure. More generally, our results provide a quantitative molecular-level basis to rationalize the experimental observation that bacteria adapted to live under high hydrostatic pressure in the deep sea are characterized by higher ratios of unsaturated fatty acids in the lipid membrane. ${ }^{25-28}$ Future work will involve simulations of more complex systems, especially including membrane proteins, aimed at quantifying high pressure effects on specific biological processes in relation to lipid diversity. 


\section{Acknowledgement}

This project made use of time on ARCHER granted via the UK High-End Computing Consortium for Biomolecular Simulation, HECBioSim (http://hecbiosim.ac.uk), supported by EPSRC (grant no. EP/L000253/1). WD acknowledges financial support by the China Scholarship Council.

\section{Supporting Information Available}

Individual electron density profiles for lipids and water, headgroup orientation data, hydrogen bonds data.

\section{References}

(1) Ritz, M.; Tholozan, J.; Federighi, M.; Pilet, M. Physiological damages of Listeria monocytogenes treated by high hydrostatic pressure. Int. J. Food. Microbiol. 2002, 79, 47-53.

(2) Funamoto, S.; Nam, K.; Kimura, T.; Murakoshi, A.; Hashimoto, Y.; Niwaya, K.; Kitamura, S.; Fujisato, T.; Kishida, A. The use of high-hydrostatic pressure treatment to decellularize blood vessels. Biomaterials 2010, 31, 3590-3595.

(3) Yang, B.; Shi, Y.; Xia, X.; Xi, M.; Wang, X.; Ji, B.; Meng, J. Inactivation of foodborne pathogens in raw milk using high hydrostatic pressure. Food Control 2012, 28, 273-278.

(4) Allen, E. E.; Facciotti, D.; Bartlett, D. H. Monounsaturated but not polyunsaturated fatty acids are required for growth of the deep-sea bacterium Photobacterium profundum SS9 at high pressure and low temperature. Appl. Environ. Microb. 1999, 65, $1710-1720$.

(5) Gaspar, L. P.; Silva, A. C.; Gomes, A. M.; Freitas, M. S.; Bom, A. P. A.; Schwarcz, W. D.; Mestecky, J.; Novak, M. J.; Foguel, D.; Silva, J. L. Hydrostatic 
pressure induces the fusion-active state of enveloped viruses. J. Biol. Chem. 2002, 277, $8433-8439$.

(6) Picard, A.; Daniel, I. Pressure as an environmental parameter for microbial life - A review. Biophysical Chemistry 2013, 183, 30 - 41.

(7) Simonato, F.; Campanaro, S.; Lauro, F. M.; Vezzi, A.; D’Angelo, M.; Vitulo, N.; Valle, G.; Bartlett, D. H. Piezophilic adaptation: a genomic point of view. Journal of biotechnology 2006, 126, 11-25.

(8) Huang, H. W.; Lung, H. M.; Yang, B. B.; Wang, C. Y. Responses of microorganisms to high hydrostatic pressure processing. Food Control 2014, 40, 250-259.

(9) Winter, R.; Dzwolak, W. Exploring the temperature-pressure configurational landscape of biomolecules: from lipid membranes to proteins. Phil. Trans. R. Soc. A 2005, 363, $537-563$.

(10) Chau, P. New insights into the molecular mechanisms of general anaesthetics. Br. J. Pharmacol .y 2010, 161, 288-307.

(11) Cherezov, V. Lipidic cubic phase technologies for membrane protein structural studies. Curr. Opin. Struct. Biol. 2011, 21, $559-566$.

(12) Purushothaman, S.; Cicuta, P.; Ces, O.; Brooks, N. J. Influence of high pressure on the bending rigidity of model membranes. J. Phys. Chem. B 2015, 119, 9805-9810.

(13) Kohli, A. G.; Kierstead, P. H.; Venditto, V. J.; Walsh, C. L.; Szoka, F. C. Designer lipids for drug delivery: from heads to tails. Journal of Controlled Release 2014, 190, $274-287$.

(14) Knorr, D.; Heinz, V.; Buckow, R. High pressure application for food biopolymers. Biochim. Biophys. Acta 2006, 1764, 619-631. 
(15) Norton, T.; Sun, D. Recent advances in the use of high pressure as an effective processing technique in the food industry. Food and Bioprocess Technology 2008, 1, 2-34.

(16) Rivalain, N.; Roquain, J.; Demazeau, G. Development of high hydrostatic pressure in biosciences: Pressure effect on biological structures and potential applications in Biotechnologies. Biotechnology advances 2010, 28, 659-672.

(17) Skanes, I. D.; Stewart, J.; Keough, K. M. W.; Morrow, M. R. Effect of chain unsaturation on bilayer response to pressure. Phys. Rev. E 2006, 74, 051913.

(18) Brooks, N. J.; Ces, O.; Templer, R. H.; Seddon, J. M. Pressure effects on lipid membrane structure and dynamics. Chem. Phys. Lipids 2011, 164, 89-98.

(19) Winter, R.; Jeworrek, C. Effect of pressure on membranes. Soft Matter 2009, 5, 31573173.

(20) Brooks, N. J. Pressure effects on lipids and bio-membrane assemblies. IUCrJ 2014, 1, $470-477$.

(21) Daniel, I.; Oger, P.; Winter, R. Origins of life and biochemistry under high-pressure conditions. Chem. Soc. Rev. 2006, 35, 858-875.

(22) Kulkarni, C. V.; Ces, O.; Templer, R. H.; Seddon, J. M. Pressure effects on a proteinlipid model membrane. Soft Matter 2013, 9, 6525-6531.

(23) Suladze, S.; Cinar, S.; Sperlich, B.; Winter, R. Pressure modulation of the enzymatic activity of phospholipase A2, a putative membrane-associated pressure sensor. J. Am. Chem. Soc. 2015, 13\%, 12588-12596.

(24) Mrozik, A.; Piotrowskaseget, Z.; Labuzek, S. Cytoplasmic bacterial membrane response to environmental perturbations. Pol. J. Environ. Stud. 2004, 13, 487.

(25) DeLong, E. F.; Yayanos, A. A. Adaptation of the membrane lipids of a deep-sea bacterium to changes in hydrostatic pressure. Science 1985, 228, 1101-1103. 
(26) Kamimura, K.; Fuse, H.; Takimura, O.; Yamaoka, Y. Effects of growth pressure and temperature on Fatty Acid composition of a barotolerant deep-sea bacterium. Appl. Environ. Microb. 1993, 59, 924-6.

(27) Bartlett, D. Pressure effects on in vivo microbial processes. Biochim. Biophys. Acta 2002, 1595, 367-381.

(28) Pond, D. W.; Tarling, G. A.; Mayor, D. J. Hydrostatic pressure and temperature effects on the membranes of a seasonally migrating marine copepod. Plos One 2013, 9, e111043-e111043.

(29) Zhu, F.; Tajkhorshid, E.; Schulten, K. Pressure-induced water transport in membrane channels studied by molecular dynamics. Biophys. J. 2002, 83, 154-160.

(30) Trzesniak, D.; Lins, R. D.; van Gunsteren, W. F. Protein under pressure: molecular dynamics simulation of the arc repressor. Proteins: Structure, Function, and Bioinformatics 2006, 65, 136-144.

(31) Thomas, M.; Corry, B. A computational assessment of the permeability and salt rejection of carbon nanotube membranes and their application to water desalination. Phil. Trans. R. Soc. A 2016, 374, 20150020.

(32) Chen, R.; Poger, D.; Mark, A. E. Effect of high pressure on fully hydrated DPPC and POPC bilayers. J. Phys. Chem. B 2010, 115, 1038-1044.

(33) Lai, K.; Wang, B.; Zhang, Y.; Zhang, Y. High pressure effect on phase transition behavior of lipid bilayers. Phys. Chem. Chem. Phys. 2012, 14, 5744-5752.

(34) Shearman, G.; Ces, O.; Templer, R.; Seddon, J. Inverse lyotropic phases of lipids and membrane curvature. J. Phys.: Condens. Matter 2006, 18, S1105.

(35) Wang, L. Measurements and implications of the membrane dipole potential. Annual review of biochemistry 2012, 81, 615-35. 
(36) Mouritsen, O. G. Life - As a Matter of Fat. The Emerging Science of Lipidomics, 1st ed.; Springer: Berlin, 2005.

(37) Templer, R. H.; Castle, S. J.; Curran, A. R.; Rumbles, G.; Klug, D. R. Sensing isothermal changes in the lateral pressure in model membranes using di-pyrenyl phosphatidylcholine. Faraday Discuss. 1998, 111, 41-53.

(38) Kamo, T.; Nakano, M.; Kuroda, Y.; Handa, T. Effects of an amphipathic $\alpha$-helical peptide on lateral pressure and water penetration in phosphatidylcholine and monoolein mixed membranes. J. Phys. Chem. B 2006, 110, 24987-24992.

(39) Ollila, O. H. S.; Vattulainen, I. In Molecular Simulations and Biomembranes: From Biophysics to Function; Sansom, M. S. P., Biggin, P. C., Eds.; The Royal Society of Chemistry, 2010; pp 26-55.

(40) van den Brink-van der Laan, E.; Killian, J. A.; de Kruijff, B. Nonbilayer lipids affect peripheral and integral membrane proteins via changes in the lateral pressure profile. Biochim. Biophys. Acta 2004, 1666, 275-288.

(41) Marsh, D. Lateral pressure profile, spontaneous curvature frustration, and the incorporation and conformation of proteins in membranes. Biophys. J. 2007, 93, $3884-$ 3899.

(42) Orsi, M.; Essex, J. W. In Molecular Simulations and Biomembranes: From Biophysics to Function; Sansom, M. S. P., Biggin, P. C., Eds.; The Royal Society of Chemistry, 2010; pp 76-90.

(43) Orsi, M.; Essex, J. W. Permeability of drugs and hormones through a lipid bilayer: insights from dual-resolution molecular dynamics. Soft Matter 2010, 6, 3797-3808.

(44) Gruner, S. M. Intrinsic curvature hypothesis for biomembrane lipid composition: a role for nonbilayer lipids. Proc. Natl. Acad. Sci. U.S.A. 1985, 82, 3665-3669. 
(45) Bezrukov, S. M. Functional consequences of lipid packing stress. Curr. Opin. Colloid Interface Sci. 2000, 5, 237-243.

(46) Orsi, M.; Noro, M. G.; Essex, J. W. Dual-resolution molecular dynamics simulation of antimicrobials in biomembranes. J. R. Soc. Interface 2011, 8, 826-841.

(47) Dreyer, J.; Zhang, C.; Ippoliti, E.; Carloni, P. Role of the membrane dipole potential for proton transport in gramicidin A embedded in a DMPC bilayer. J. Chem. Theory Comput. 2013, 9, 3826-3831.

(48) Klauda, J. B.; Venable, R. M.; Freites, J. A.; OConnor, J. W.; Tobias, D. J.; Mondragon-Ramirez, C.; Vorobyov, I.; MacKerell Jr, A. D.; Pastor, R. W. Update of the CHARMM all-atom additive force field for lipids: validation on six lipid types. J. Phys. Chem. B 2010, 114, 7830-7843.

(49) Zhuang, X.; Makover, J. R.; Im, W.; Klauda, J. B. A systematic molecular dynamics simulation study of temperature dependent bilayer structural properties. Biochim. Biophys. Acta 2014, 1838, 2520-2529.

(50) Ding, W.; Palaiokostas, M.; Wang, W.; Orsi, M. Effects of lipid composition on bilayer membranes quantified by all-atom molecular dynamics. J. Phys. Chem. B 2015, 119, 15263-15274.

(51) Berendsen, H. J.; van der Spoel, D.; van Drunen, R. GROMACS: a message-passing parallel molecular dynamics implementation. Comput. Phys. Comm. 1995, 91, 43-56.

(52) Darden, T.; York, D.; Pedersen, L. Particle mesh Ewald: An N log (N) method for Ewald sums in large systems. J. Chem. Phys. 1993, 98, 10089-10092.

(53) Miyamoto, S.; Kollman, P. A. SETTLE: an analytical version of the SHAKE and RATTLE algorithm for rigid water models. J. Comput. Chem. 1992, 13, 952-962. 
(54) Hess, B.; Bekker, H.; Berendsen, H. J.; Fraaije, J. G. LINCS: a linear constraint solver for molecular simulations. J. Comput. Chem. 1997, 18, 1463-1472.

(55) Parrinello, M.; Rahman, A. Polymorphic transitions in single crystals: A new molecular dynamics method. J. Appl. Phys. 1981, 52, 7182-7190.

(56) Bussi, G.; Donadio, D.; Parrinello, M. Canonical sampling through velocity rescaling. J. Chem. Phys. 2007, 126, 014101.

(57) Paschek, D. Temperature dependence of the hydrophobic hydration and interaction of simple solutes: An examination of five popular water models. J. Chem. Phys. 2004, 120, 6674-6690.

(58) Ghosh, T.; Garca, A. E.; Garde, S. Molecular Dynamics Simulations of Pressure Effects on Hydrophobic Interactions. Journal of the American Chemical Society 2001, 123, 10997-11003, PMID: 11686704.

(59) Ding, W.; Palaiokostas, M.; Orsi, M. Stress testing the ELBA water model. Mol. Simul. 2016, 42, 337-346.

(60) Jambeck, J. P.; Lyubartsev, A. P. An extension and further validation of an all-atomistic force field for biological membranes. J. Chem. Theory Comput. 2012, 8, 2938-2948.

(61) Orsi, M.; Essex, J. W. Physical properties of mixed bilayers containing lamellar and nonlamellar lipids: insights from coarse-grain molecular dynamics simulations. Faraday Discuss. 2013, 161, 249-272.

(62) Vanegas, J. M.; Torres-Sánchez, A.; Arroyo, M. Importance of force decomposition for local stress calculations in biomembrane molecular simulations. J. Chem. Theory Comput. 2014, 10, 691-702.

(63) Torres-Sánchez, A.; Vanegas, J. M.; Arroyo, M. Examining the mechanical equilibrium of microscopic stresses in molecular simulations. Phys. Rev. Lett. 2015, 114, 258102. 
(64) Flyvbjerg, H.; Petersen, H. G. Error estimates on averages of correlated data. J. Chem. Phys. 1989, 91, 461-466.

(65) Rapaport, D. C. The art of molecular dynamics simulation; Cambridge university press, 2004.

(66) Orsi, M. Comparative assessment of the ELBA coarse-grained model for water. Mol. Phys. 2014, 112, 1566-1576.

(67) Piggot, T. J.; Pineiro, A.; Khalid, S. Molecular dynamics simulations of phosphatidylcholine membranes: a comparative force field study. J. Chem. Theory Comput. 2012, 8, 4593-4609.

(68) Nagle, J. F.; Tristram-Nagle, S. Structure of lipid bilayers. Biochim. Biophys. Acta 2000, 1469, $159-195$.

(69) Kučerka, N.; Tristram-Nagle, S.; Nagle, J. F. Structure of fully hydrated fluid phase lipid bilayers with monounsaturated chains. J. Membr. Biol. 2006, 208, 193-202.

(70) Martinac, B.; Hamill, O. P. Gramicidin A channels switch between stretch activation and stretch inactivation depending on bilayer thickness. Proc. Natl. Acad. Sci. U.S.A. 2002, 99, 4308-4312.

(71) Brown, A.; Skanes, I.; Morrow, M. R. Pressure-induced ordering in mixed-lipid bilayers. Phys. Rev. E 2004, 69, 011913.

(72) Filippov, A.; Orädd, G.; Lindblom, G. The effect of cholesterol on the lateral diffusion of phospholipids in oriented bilayers. Biophys. J. 2003, 84, 3079-3086.

(73) Gaede, H. C.; Gawrisch, K. Lateral diffusion rates of lipid, water, and a hydrophobic drug in a multilamellar liposome. Biophys. J. 2003, 85, 1734-1740.

(74) Ollila, S.; Hyvönen, M. T.; Vattulainen, I. Polyunsaturation in lipid membranes: dynamic properties and lateral pressure profiles. J. Phys. Chem. B 2007, 111, 3139-3150. 
(75) Ollila, O. H. S.; Rog, T.; Karttunen, M.; Vattulainen, I. Role of sterol type on lateral pressure profiles of lipid membranes affecting membrane protein functionality: comparison between cholesterol, desmosterol, 7-dehydrocholesterol and ketosterol. J. Struct. Biol. 2007, 159, 311-323.

(76) Orsi, M.; Essex, J. W. The ELBA force field for coarse-grain modeling of lipid membranes. PLoS One 2011, 6, e28637.

(77) Cevc, G.; Marsh, D. Phospholipid bilayers: physical principles and models; Wiley, 1987.

(78) Mukhin, S. I.; Baoukina, S. Analytical derivation of thermodynamic characteristics of lipid bilayer from a flexible string model. Phys. Rev. E 2005, 71, 061918.

(79) Orsi, M.; Haubertin, D. Y.; Sanderson, W. E.; Essex, J. W. A quantitative coarse-grain model for lipid bilayers. J. Phys. Chem. B 2008, 112, 802-815.

(80) Gawrisch, K.; Holte, L. L. NMR investigations of non-lamellar phase promoters in the lamellar phase state. Chem. Phys. Lipids 1996, 81, 105-116.

(81) Szleifer, I.; Kramer, D.; Ben-Shaul, A.; Gelbart, W. M.; Safran, S. A. Molecular theory of curvature elasticity in surfactant films. J. Chem. Phys. 1990, 92, 6800-6817.

(82) Ben-Shaul, A. In Structure and Dynamics of Membranes; Lipowsky, R., Sackmann, E., Eds.; Elsevier: Amsterdam, 1995; pp 359-401.

(83) Rawicz, W.; Olbrich, K.; McIntosh, T.; Needham, D.; Evans, E. Effect of chain length and unsaturation on elasticity of lipid bilayers. Biophys. J. 2000, 79, 328-339.

(84) Pan, J.; Tristram-Nagle, S.; Kučerka, N.; Nagle, J. F. Temperature dependence of structure, bending rigidity, and bilayer interactions of dioleoylphosphatidylcholine bilayers. Biophys. J. 2008, 94, 117-124.

(85) Orsi, M.; Michel, J.; Essex, J. W. Coarse-grain modelling of DMPC and DOPC lipid bilayers. J. Phys.: Condens. Matter 2010, 22, 155106. 
(86) Chen, Z.; Rand, R. P. The influence of cholesterol on phospholipid membrane curvature and bending elasticity. Biophys. J. 1997, 73, 267-276.

(87) Cantor, R. S. The influence of membrane lateral pressures on simple geometric models of protein conformational equilibria. Chem. Phys. Lipids 1999, 101, 45-56.

(88) Attard, G. S.; Templer, R. H.; Smith, W. S.; Hunt, A. N.; Jackowski, S. Modulation of CTP: phosphocholine cytidylyltransferase by membrane curvature elastic stress. Proc. Natl. Acad. Sci. U.S.A. 2000, 97, 9032-9036.

(89) Polyansky, A. A.; Volynsky, P. E.; Nolde, D. E.; Arseniev, A. S.; Efremov, R. G. Role of lipid charge in organization of water/lipid bilayer interface: Insights via computer simulations. J. Phys. Chem. B 2005, 109, 15052-15059.

(90) Siu, S. W.; Vácha, R.; Jungwirth, P.; Böckmann, R. A. Biomolecular simulations of membranes: physical properties from different force fields. J. Chem. Phys. 2008, 128, 125103. 


\section{Graphical TOC Entry}

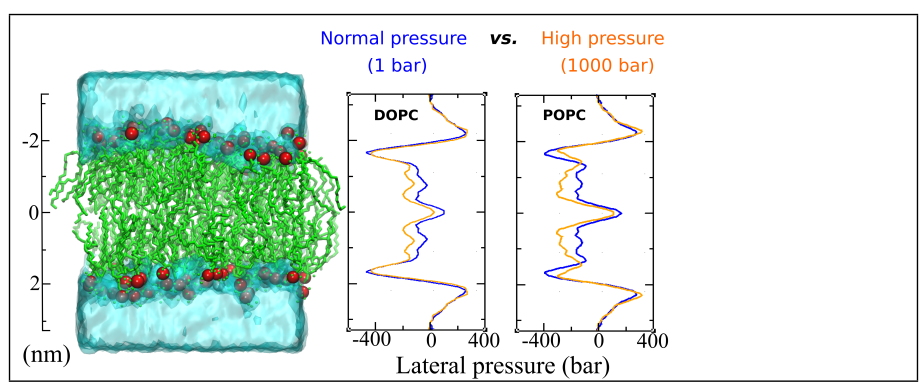

14

15

16

17

18

19

20

21

22

23

24

25

26

27

28

29

30

31

32

33

34

35

36

37

38

39

40

41

42

43

44

45

46

47

48

49

50

51

52

53

54

55

56

57

58

59

60 